\title{
Testing of the DR Method for Image Sharpness Determination
}

\author{
M Matthews ${ }^{1}$ and $\mathrm{J} \mathrm{Shah}^{2}$ \\ 1. AWE, Aldermaston, Reading, UK \\ 2. Visiting Fellow, University of Bristol, Bristol, UK
}

The current practice of specifying instrument resolution in SEM, of measuring the smallest visible gap between gold particles on a carbon substrate is, at best, not reliably repeatable and, at worst, dependant on the 'eye of the beholder'.

The International Standards Organisation Technical Committee on Microbeam Analysis, ISO/TC202, is developing a standard method for the quantitative determination of image sharpness in electron micrographs. Of the various procedures that have been appraised to date for their suitability as a standard the Derivative (DR) Method shows promise.

The procedure, shown diagrammatically in Figure 1, is to first identify edges in the image area and draw profiles of predetermined length perpendicular to these. In the software version used for this study profiles with insufficient contrast are rejected (shown in red in Figure 1). The average of the slope parameters of an error function fitted through each of the identified profiles gives the sharpness value, $s$ for the image.

After initial testing of a set of eleven images distributed to the technical committee working group nine images were selected for detailed testing by the authors. From the shape of the two dimensional angular distribution plot of $\sigma$ (where $\sigma=S / \sqrt{ } 2$ ) it was possible to determine the degree of the noise distribution in the micrographs. The degree of astigmatism, if present was also detectable. Sharpness values were derived for each, varying individual input parameters, to determine the relative sensitivities of the method. The following key points were identified:

- Although the procedure was able to extract edge profiles from a range of sample types it could not be used on every sample. For example two sample images failed to derive meaningful sharpness values.

- All the images tested showed steady sharpness values for a given set of parameters when more than $100-200$ edges were averaged. This was also the case for micrographs with considerable noise.

- The computer program for calculating sharpness allows different input values of several parameters such as distance between the pixels processed, the profile (edge) length, number of edges (Figure 2) etc. The profile length parameter showed the greatest sensitivity, changing the derived sharpness value by up to $\pm 15 \%$ in the images tested.

- The sharpness value reached a steady state with increasing profile length for the most of the images (Figure 2), although for one image this was not the case

- It was not visually apparent which images would return convergent value of sharpness.

Of the methods so far tested by the ISO technical committee the DR method shows the most promise. It can be applied to a range of image/sample types and generates a stable sharpness value with only 100 200 edges. However, checks need to be included in the procedure to test for the sensitivity to profile length. 

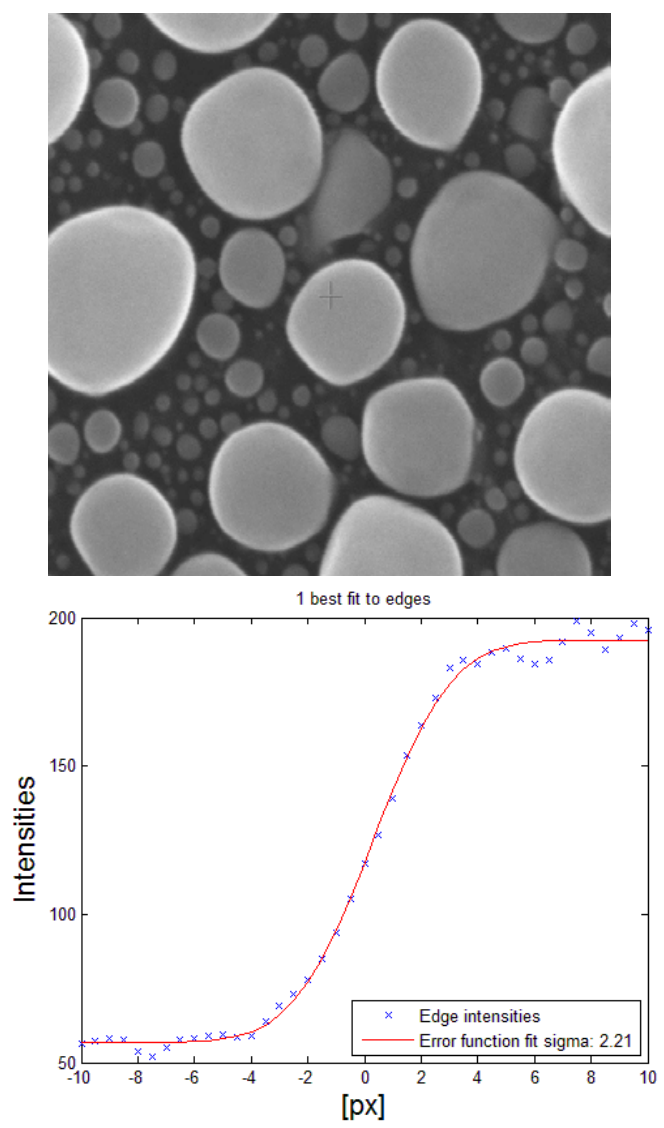
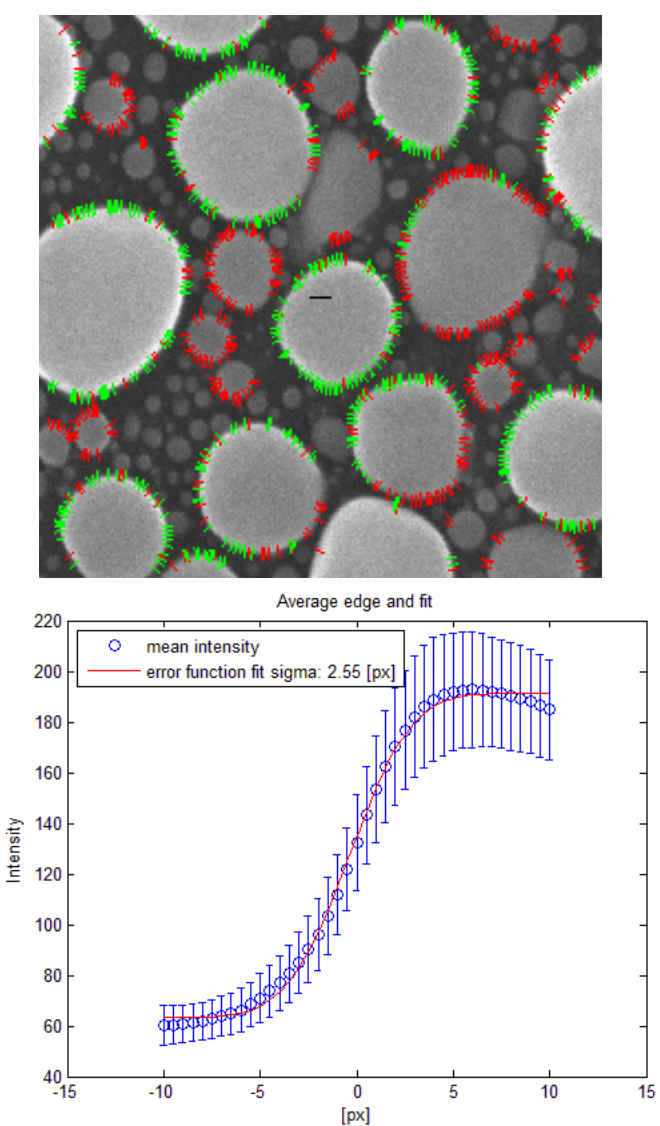

Figure 1 Image as acquired (upper left), edges identified and profiles drawn (upper right), error function fitted through each profile (lower left), average profile (lower right) derives sharpness value.
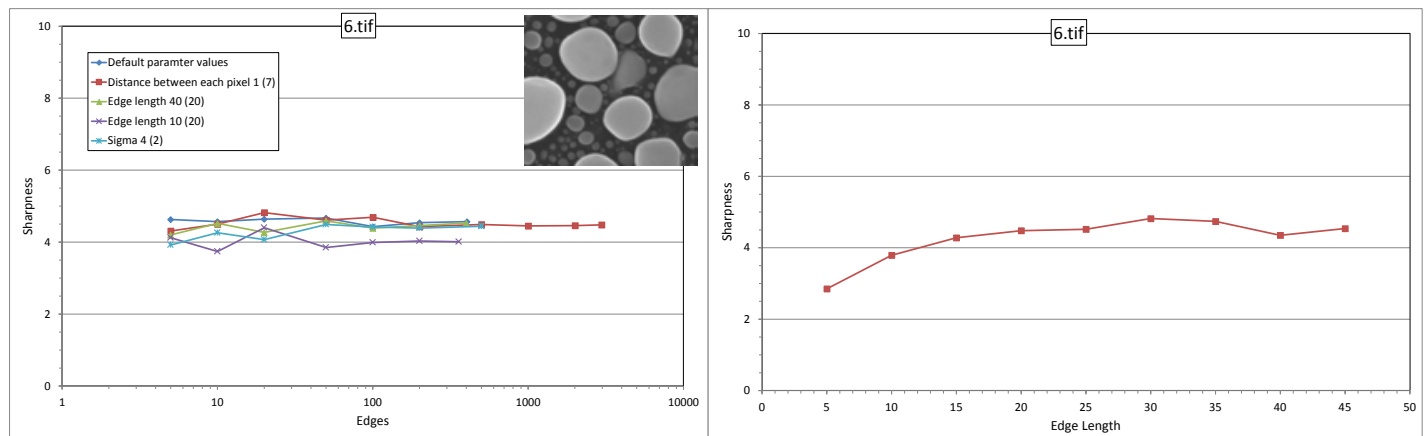

Figure 2 Sharpness results for a 'well behaved' image. 